\title{
A Case of Hypofibrinogenemia Presenting with Submental Hamatoma
}

\author{
Mujahida Rahman', Naseeb Muhammad Irshadullah², Masuda Begum ${ }^{3}$ \\ ${ }^{1}$ Medical Officer, ${ }^{2}$ Resident, ${ }^{3}$ Professor, Department of Hamatology, BSMMU
}

\begin{abstract}
:
Hereditary hypofibrinogenemia is a rare disease and the usual presentation is difficult to stop bleeding or hamatoma in the muscle or intracranial space after injury. There may also be adverse pregnancy outcome, and increased tendency to thrombosis. The usual measure is replacement of fibrinogen. Here a case of this rare disease with unusual presentation is reported, and the special aspects of management are discussed.
\end{abstract}

Keywords: fibrinogen, hypofibrinogenemia, afibrinogenemia, haematoma

[BSMMU J 2014; $7(1): 65-67]$

\section{Introduction:}

Inherited disorders of fibrinogen affect either the quantity (afibrinogenemia and hypofibrinogenemia) or the quality (dysfibrinogenemia) of the circulating fibrinogen or both (hypodysfibrinogenemia). Most often, patients with congenital fibrinogen disorders suffer from a bleeding diathesis but paradoxically may undergo severe thrombotic episodes which are usually with dysfibrinogenemia or with fibrinogen replacement ${ }^{1}$. Pregnancy loss is another common clinical complication. Even in specialized laboratories, the precise diagnosis of some fibrinogen disorders may be challenging. Characterization of the molecular defect(s) is important as it provides a more accurate diagnosis, may enable prenatal diagnosis, will help elaborate a diagnostic strategy, and may distinguish in some cases those patients at risk of thrombosis rather than bleeding. However, the phenotype-genotype correlation is not easy to establish, and global hemostasis assays may provide a better evaluation of the patient's hemostatic state $^{2}$.

Fibrinogen is a $340-\mathrm{kDa}$ plasma protein that circulates at a concentration of 1.5 to $3.5 \mathrm{mg} / \mathrm{ml}$. It is the clotting factor I of coagulation pathway, and the precursor of the insoluble end product of that pathway, i.e. fibrin. Without fibrinogen, not only clotting is impossible but also some

Address for Correspondence: Dr. Mujahida Rahman

Medical Officer, Department of Hematology, BSMMU, Dhaka.

Mob.01712629666,E-mail: dr.mujahida21@gmail.com defect in platelet function leads to prolonged bleeding time in one third of the patients ${ }^{3}$. Absence of fibrinogen causes less severe bleeding than what would be expected, probably because of presence of functional vWF, which allows platelet adhesion and aggregation, with the formation of loose thrombi even in the absence of fibrinogen ${ }^{4}$. The inheritance pattern of afibrinogenemia is autosomal recessive in nature, and many reported cases are the result of consanguineous relationships between asymptomatic parents with symptomatic homozygote offspring.

In patients with afibrinogenemia, life-threatening hemorrhages do occur, but in many situations the bleeding is not as severe as is seen in hemophilia. The diagnosis is often made early in infancy, when prolonged umbilical stump bleeding occurs ${ }^{6}$. A major cause of death is intracranial hemorrhage during infancy or childhood ${ }^{7}$. The clinical manifestations include mucosal membrane bleeding, such as epistaxis, menorrhagia, or gastrointestinal hemorrhage. A review of the cases entered into the North American Rare Bleeding Disorder Registry indicated that most bleeding events were triggered by trauma, with only 20 to $30 \%$ occurring spontaneously ${ }^{8}$. Patients with congenital hypofibrinogenemia do not typically have any spontaneous bleeding unless the fibrinogen level is $<50 \mathrm{mg} / \mathrm{dl}$.

A-or hypofibrinogenemia patients typically have prolonged clotting time $(\mathrm{CT})$, prothrombin time (PT), partial thromboplastin time (PTT), thrombin clotting time, and reptilase time which are usually corrected when 
Patient plasma is mixed with normal plasma. Bleeding time (BT) may also be prolonged and a possible explanation is mentioned earlier. When the fibrinogen levels are low, the acquired quantitative disorder of fibrinogen, namely, liver disease or disseminated intravascular coagulation, should be excluded. It is also reported in patients getting L-asparaginase for acute lymphoblastic leukemia $(\mathrm{ALL})^{9}$ or an antithymocyte globulin (ATG) and corticosteroids for aplastic anemia (AA) ${ }^{10}$. Increased fibrinogen clearance was observed with massive hemorrhage, major surgery, and advanced pregnancy ${ }^{11}$.

Replacement of the deficient or abnormal fibrinogen with frozen plasma, cryoprecipitate, or fibrinogen concentrate has been found to be effective and is indicated for any episode of acute active bleeding, preoperatively, and in pregnant patients. Fibrinogen levels between 50 and 100 $\mathrm{mg} / \mathrm{dl}$ are usually adequate for normal hemostasis, levels $>100 \mathrm{mg} / \mathrm{dl}$ are recommended for maintenance during pregnancy, based on empiric clinical observations and $100-200 \mathrm{mg} / \mathrm{dl}$ for surgical procedures ${ }^{11}$. Each bag of cryoprecipitate contains $250 \mathrm{mg}$ of fibrinogen, and one bag of cryoprecipitate typically raises plasma fibrinogen level of an adult by $10 \mathrm{mg} / \mathrm{dl}$; thus 5 to 10 bags of cryoprecipitate are usually sufficient in the average adult patient. Because the fractional catabolic rate of fibrinogen is $25 \%$ per day, acute-care patients should receive one third of their loading dose daily for as long as fibrinogen support is desired ${ }^{2}$.

\section{Case report:}

A 12-year-old school girl from a poor family of Bijoynagar, Brahman Baria, was admitted into the Department of Haematology, Bangabandhu Sheikh Mujib Medical University (BSMMU) on 11 July 2012 with uncontrollable bleeding from mouth for 2 days and a swelling in the floor of the mouth for the same duration. She gave history of prolong bleeding and also bleeding from umbilical stump 4 days after birth. She had no family history of such bleeding or consanguineous marriage of parents. For unusual bleeding she was admitted to Dhaka Shishu Hospital in 2003 and several times treated with $\mathrm{I} / \mathrm{V}$ tranexamic acid. On admission she had no other bleeding points or history of trauma to mouth or face. Examination of the mouth showed a hematoma in the floor of the mouth that pushed the tongue backwards and blood tinged saliva. The hematoma caused difficulty with speech and swallowing of food and saliva. She was or icteric; there was no lymphadenopathy, organomegaly or bony tenderness. Immediately after admission blood was sent for baseline investigations including coagulation profile. Her PT was $40 \mathrm{sec}$ (control $11.8 \mathrm{sec}$ ), APTT was $88 \mathrm{sec}$ (control $28.8 \mathrm{sec}$ ), plasma fibrinogen level $50 \mathrm{mg} / \mathrm{dl}$, D-dimer $>10.0 \mathrm{mg} / \mathrm{L}, \mathrm{Hb}$ level $11.3 \mathrm{gm} / \mathrm{dl}$, Platelet $300,000 / \mathrm{mm}^{3}$, WBC9,000/ $\mathrm{mm}^{3}$ (neutrophil 54\%). PBF showed microcytic hypochromic anemia which might be due to recurrent bleeding. RBS, SGPT, S. creatinine and S. albumin were within normal range. After transfusion of 12 units of FFP over 4 days of hospital stay her bleeding stopped and repeat plasma fibrinogen level was 194 $\mathrm{mg} / \mathrm{dl}$.

\section{Discussion:}

Inherited a- or hypofibrinogenemia is a rare disease, with an estimated frequency of one in a million individuals ${ }^{18}$. The usual presentation is discussed above, which includes excessive tendency to bleeding after trauma but spontaneous bleeding at $50 \mathrm{mg} / \mathrm{dl}$ level of fibrinogen is very uncommon. So, this young girl may be a case of hypodysfibrinogenemia, but functional assay of fibrinogen was not available that time at BSMMU. There are reports of hematomas under skin or within muscles, but submental hematoma is rare feature. For financial constraint and unavailability fibrinogen concentrate could not be given, but the girl responded well to FFP and I/V tranexamic acid. The special caution taken should be for menarche, menstruation and pregnancy. Increased incidence of firsttrimester abortion, placental abruption, and postpartum hemorrhage has been observed in patients with afibrinogenemia ${ }^{19}$. Fetuses of female afibrinogenemic patients rarely reach full term unless replacement therapy is given. Moreover, though uncommon, there may excess chance of thromboembolism if she would choose to take oral contraceptive pills to avoid pregnancies. On the other hand, intra-uterine contraceptive devices may cause traumatic per vaginal bleeding. Consanguineous marriage should also be discouraged to reduce the chance of disease in the offspring, and if available prenatal diagnosis may be 
diagnosis may be advised.

\section{Conclusion:}

In any bleeding diathesis, the recommended algorithm should be followed, and this will allow even rare cases to be diagnosed at the early manifestations. Otherwise, the diagnosis might be delayed, like that happened to this girl. Many hematological diseases require sophisticated laboratory facilities for complete evaluation, but judicious use of available resources will help us to diagnosis the disease, and thereby educate the patient properly.

\section{References:}

1. Mosesson MW. Hereditary abnormalities of fibrinogen. In: Beutler E, Lichtman MA, Coller BS, et al., eds. Williams hematology, 6th ed. New York: McGraw-Hill, 2001:1659-1671.

2. de Moerloose P, Neerman-Arbez M. Congenital fibrinogen disorders. Semin Thromb Hemost 2009 Jun;35(4):356-66.

3. Cattaneo M, Bettega D, Lombardi E, Sustained correction of the bleeding time in an afibrinogenemic patient after infusion of fresh frozen plasma. Br J Haematol 1992;82:388-390.

4. Ni H, Denis CV, Subbarao S, Persistence of platelet thrombus formation in arterioles of mice lacking both von Willebrand factor and fibrinogen. J Clin Invest 2000;106:385-392.
5. Moen JL, Lord ST. Afibrinogenemias and dyfibrinogenemias. In: Colman RW, Marder VJ, Clowes AW, et al., eds. Hemostasis and thrombosis: Basic principles and clinical practice. Philadelphia: Lippincott Williams \& Wilkins, 2006:939-952.

6. Bolton-Maggs PHB, Perry DJ, Chalmers EA, The rare coagulation disorders - review with guidelines for management from the United Kingdom Haemophilia Centre Doctors' Organisation. Haemophilia 2004;10:593-628.

7. Fried K, Kaufman S. Congenital afibrinogenemia in 10 offspring of uncle-niece marriages. Clin Genet 1980;17:223-227.

8. Acharya SS, Coughlin A, Dimichele DM, Rare Bleeding Disorder Registry: Deficiencies of factors II, V, VII, X, XIII, fibrinogen and dysfibrinogenemias. J Thromb Haemost 2003;2:248-256.

9. Ramsay NKC, Coccia PF, Krivit W, The effect of L-asparaginase on plasma coagulation factors in acute lymphoblastic leukemia. Cancer 1977;40:1398-1401.

10. Fischer M, Lechner K, Hinterberger W, Deficiency of fibrinogen and factor VII following treatment of severe aplastic anaemia with anti-thymocyte globulin and high-dose methylprednisolone. Scand J Haematol 1985;34:312-316.

11. Bornikova L, Peyvandi F, Allen G, Bernstein J, Manco-Johnson MJ. Fibrinogen replacement therapy for congenital fibrinogen deficiency. J Thromb Haemost. 2011 Sep;9(9):1687-704.

12. Neerman-Arbez M. The molecular basis of inherited afibrinogenaemia. Thromb Haemost 2001;86:154-163.

13. Goodwin TM. Congenital hypofibrinogenemia in pregnancy. Obstet Gynecol Surv 1989;44:157-161. 